\title{
COMPLEXATION OF POLY(ETHYLENE OXIDE) WITH POLY(ACRYLIC ACID-CO-HYDROXYETHYL METHACRYLATE)S
}

\author{
MAARTEN J. KRUPERS*, FRED J. VAN DER GAAG and JAN FEIJEN $\dagger$ \\ Department of Chemical Technology and Institute for Biomedical Technology, University of Twente, \\ P.O. Box 217, 7500 AE Enschede, The Netherlands
}

(Received 8 December 1994; accepted in final form 27 June 1995)

\begin{abstract}
Random acrylic acid/hydroxyethyl methacrylate (AA/HEMA) copolymers were synthesized to study the influence of non-ionic (HEMA) structure defects introduced in poly(acrylic acid) on the complex formation with PEO $\left(\bar{M}_{\mathrm{w}} 23200\right)$ at $\mathrm{pH} 3-3.5$ in aqueous solution. Complex formation as studied with potentiometric titration (concentration $0.01-0.03 \mathrm{M}$ monomer residues) was increasingly impeded when the molar ratio of HEMA to AA units in the polymers increased. Copolymers of AA and HEMA with molar ratios $30: 1,10: 1$ and $3: 1$ were used in this study. It was shown that in the copolymers the number of carboxylic acid groups taking part in complexation decreased with increasing amounts of HEMA in the copolymer. However, with increasing amounts of structure defects the amount of PEO per AA residue (at equilibrium complexation) increased, suggesting less efficient packing of PEO on the acrylic copolymer chain. Copyright $\mathbb{C} 1996$ Elsevier Science Ltd
\end{abstract}

\section{INTRODUCTION}

It is well known that intermacromolecular complexes can be formed by mixing solutions of two complementary polymers [1-5]. These complexes are stabilized by electrostatic interactions (polyanionpolycation) or hydrogen bonds (polyacid-polybase) between the polymers involved or by hydrophobic interactions between the polymers in an aqueous surrounding. Mixing solutions of poly(acrylic acid) (PAA) and poly(ethylene oxide) (PEO) under the proper conditions results in the formation of complexes based on hydrogen bonding.

Only a few articles on the use of copolymers for the formation of such complexes have been published [6-13]. Iliopoulos et al. studied the complexation of partially neutralized PAA with PEO using potentiometry, viscometry and fluorescence polarization measurements [11-13]. The modification implied creation of structure defects by introducing ionized acrylate groups via partial neutralization with sodium hydroxide. The authors found that the occurrence of complexation depended strongly on the number of structure defects present. It was concluded that $15 \%$ ionization totally impeded complex formation. Further, it was stated that a sequence of at least seven acrylic acid units in the protonated state was necessary to show any complex formation. However, it has to be noted that non-associative behaviour is favoured by the nature of the structure defects. The negatively charged acrylate groups repulse the proton-accepting ether oxygens in the PEO chain.

*Present address: Department of Makromolekulare Chemie, Organische Chemie III, Universität UIm, Germany.

†To whom all correspondence should be addressed.
Furthermore, these structure defects are able to move along the polymer chain during the establishment of the complexation equilibrium.

More recently, Baranovsky et al. [14] showed that a functional group in the PEO chain has a pronounced influence on the complexation, depending on the type and position (in the middle of the PEO chain or on its end) of the functional group. The presence of phenol, $p$ - $t$-butylphenol, 1-naphthol, $n$ octanol or hydroquinone as a functional group was shown to increase complex stability due to negative interaction between the hydrophobic functional group in the free PEO and the solvent water as compared to the complexed PEO. In this paper the influence of non-ionic fixed structure defects in the PAA chain on the complex formation with PEO is described. Copolymers containing acrylic acid (AA) and hydroxyethyl methacrylate (HEMA) units were synthesized and the complex formation with PEO in aqueous solution at a $\mathrm{pH}$ of 3-3.5 was studied using potentiometric titration.

\section{EXPERIMENTAL PROCEDURES}

\section{Chemicals}

Toluene, ethyl acetate, methanol and $n$-hexane were chemically pure grade. 1,4-Dioxane $(99.5 \%$, Merck, Germany) was p.a. quality. Demineralized water was of ultrapure quality (typical conductivity $5.5 \times 10^{-8} \mathrm{~S} \mathrm{~cm}^{-1}$ ) as obtained from a Millipore Milli-Q water purification unit. Azo-bisisobutyronitrile (AIBN, $>98 \%$, Fluka, Switzerland) and acrylic acid (AA, 99\%, Aldrich) were used as received. Hydroxyethyl methacrylate (HEMA, BDH Chemicals, England) was purified by the method of Macret et al. [15] HEMA was brought on a Silicagel 60 (Merck) column and subsequently eluted with an ethyl acetate/toluene $75 / 25(\mathrm{v} / \mathrm{v})$ mixture. The fractions containing only HEMA, as shown by 
TLC, were collected, and the eluent was carefully removed on a rotary evaporator $\left(T<30^{\circ} \mathrm{C}\right)$.

Poly(ethylene oxide)s (PEOs) (PEO-3.4, MW 3400, Janssen Chimica, Belgium, PEO-6, MW 6000, Fluka, PEO25, MW 25,000, Merck-Schuchard, Germany) were purified by dissolution in ethanol followed by precipitation in $n$-hexane. PEO MW 1000 (PEO- I, Shell, The Netherlands) was dissolved in THF followed by precipitation in $n$-hexane. Molecular weights were determined by gel permeation chromatography-low angle laser light scattering (GPC/LALLS) in $0.1 \mathrm{M}$ acetate buffer ( $\mathrm{pH}$ 6) using a Waters Associates 510 pump, a Waters Associates differential refractometer Model R410 and three columns (Chrompack), TSK Gel type G $5000 \mathrm{PW}, \mathrm{G} 4000 \mathrm{PW}$ and $\mathrm{G} 3000 \mathrm{PW}$, connected in series. The LALLS used was a Chromatix model KMX-6 (Milton Roy). The molecular weight of the smaller PEOs was also determined using hydroxyl end-group titration according to DIN 53240.

Proton NMR spectra were recorded on a Bruker AC 250 spectrometer $\left(250 \mathrm{MHz}, 160\right.$ scans) in $\mathrm{D}_{2} \mathrm{O}$, using solutions of approximately $20 \mathrm{mg}$ polymer in $0.7 \mathrm{ml} \mathrm{D}_{2} \mathrm{O}(99.8$ atom $\% \mathrm{D})$. The HDO peak $(4.63 \mathrm{ppm})$ was used as reference.

\section{Polymerizations}

Poly(acrylic acid) (PAA) and poly(AA-co-HEMA) were synthesized by free radical polymerization. In a typical procedure, a 1 litre, three-necked round-bottomed flask, equipped with a mechanical stirrer, a thermometer and a nitrogen inlet, containing $500 \mathrm{ml}$ of water was heated to $80^{\circ} \mathrm{C}$. Then a solution of 3 mmole $(0.5 \mathrm{~g})$ of AIBN in a mixture of 0.35 mole of monomer(s) was added. After $5 \mathrm{hr}$ stirring at $80^{\circ} \mathrm{C}$ the mixture was allowed to cool to room temperature. The solvent was removed by freeze drying and the residue was purified by dissolution in methanol and precipitation in diethyl ether. The solid polymer was collected by filtration and dried in vacuo. The yield of (co)polymer after freeze drying was higher than $90 \%$.

Poly(acrylic acid) and copolymers with molar feed ratios of AA to HEMA of $30: 1,10: 1$ and $3: 1$ were prepared.

Viscosity data were used to calculate the molecular weight for PAA from the Mark-Houwink equation: $[\eta]=K \cdot \bar{M}_{\mathrm{v}}^{x}$ The values for the constants used are $K=0.076 \mathrm{ml} / \mathrm{g}$ and $\alpha=0.50$ at $30^{\circ} \mathrm{C}$ in 1,4 -dioxane [16]. Viscometry was performed using an Ubbelohde viscometer (Cannon State College PA, 100, L20) in a water bath at $30^{\circ} \mathrm{C}$.

\section{Titration experiments}

Complex formation was monitored via a potentiometric titration in which aliquots of a PEO solution were added to the solution of the acrylic acid(co)polymer. During the experiment the $\mathrm{pH}$ of the solution was recorded. In the case of the poly(AA-co-HEMA) the copolymers were dissolved in water by adding a few drops of $1 \mathrm{M} \mathrm{NaOH}$ solution. Before the titration experiment was started the $\mathrm{pH}$ was adjusted to $3.3-3.5$ by adding $\mathrm{HCl}$ solution. Care was taken to avoid precipitation of the copolymer from solution. PAA dissolved in water without the addition of $\mathrm{NaOH}$ so no adjustment of the $\mathrm{pH}$ was necessary before titration.

Titration experiments were performed in triplicate using $50 \mathrm{ml}$ of an acrylic acid (co)polymer solution (0.01, 0.02 and $0.03 \mathrm{M}$ ) to which aliquots of a $0.8 \mathrm{M}$ PEO solution were added. After each addition the $\mathrm{pH}$ was registered when a stable $\mathrm{pH}$ was obtained (approximately $2 \mathrm{~min}$ ). The concentrations of the polymers are expressed as molar concentrations of monomer residues.

\section{RESULTS AND DISCUSSION}

Because complexation is strongly dependent on the molecular weight of both poly-acid and poly-base, the molecular weights for the polymers used were
Table 1. Molecular weights of poly(ethylene oxides)

\begin{tabular}{lcc}
\hline Code & $\begin{array}{c}M_{\mathrm{n}} \text { titration } \\
(\mathrm{kg} / \text { mole })\end{array}$ & $M_{\mathrm{w}} \begin{array}{c}\text { GPC/LALLS } \\
(\mathrm{kg} / \text { mole })\end{array}$ \\
\hline PEO-1 & $1.01 \pm 0.04$ & - \\
PEO-3.4 & $3.0 \pm 0.2$ & - \\
PEO-6 & $8.2 \pm 0.3$ & 7.6 \\
PEO-25 & - & 23.2 \\
\hline
\end{tabular}

determined. In Table 1 the PEO molecular weights are given, as determined by endgroup titration and/or GPC/LALLS.

To obtain an impression about the molecular weights of the acrylic acid(co)polymers, first the molecular weight of PAA was determined by GPC/LALLS and viscometry. The molecular weight of PAA was determined to be $\bar{M}_{w}=286 \mathrm{~kg} / \mathrm{mole}$ using GPC/LALLS and $\bar{M}_{\mathrm{v}}=327 \pm 10 \mathrm{~kg} / \mathrm{mole}$ by viscometry in 1,4-dioxane. Viscometry using aqueous buffer (phosphate, $1 \mathrm{M}, \mathrm{pH} 7$ ) was performed to obtain an impression of the molecular weight of the copolymers. The intrinsic viscosities $[\eta]$ for PAA and the $30: 1,10: 1$ and $3: 1$ copolymers are $2.89,2.21,2.19$ and $1.18 \mathrm{dl} / \mathrm{g}$, respectively. Thus it may be concluded that the molecular weights of the acrylate polymers are always high enough to take part in complex formation with PEO-25 (minimum chain length for PAA ca. $500 \mathrm{~g} /$ mole, 7 monomeric units [13]).

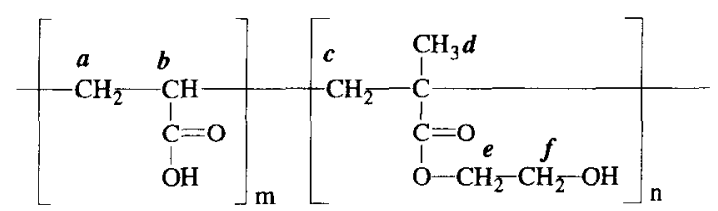

Scheme 1. Structural formulas of copolymers showing ${ }^{1} \mathrm{H}$ NMR assignments.

A ${ }^{1} \mathrm{H}-\mathrm{NMR}$ study was carried out for quantitative analysis of the overall AA to HEMA ratio in the synthesized copolymers. In Fig. 1 the ${ }^{1} \mathrm{H}-\mathrm{NMR}$ spectra of PAA and P(AA-co-HEMA)s are shown. Monomer ratios were calculated based on the $\alpha$ methyl group protons ( $1.22 \mathrm{ppm})$ in HEMA as well as on the alkyl protons in the hydroxyethyl group of HEMA (3.9 and $4.3 \mathrm{ppm}$ ), compared to the backbone methylene protons (1.3-2.1 ppm). Thus, the average ratios obtained for $\mathrm{P}$ (AA-co-HEMA)-30/1, P(AAco-HEMA)-10/1 and P(AA-co-HEMA)-3/1 were determined to be $31.5 \pm 5.7,9.0 \pm 1.7$ and $5.0 \pm 0.5$, respectively (ratio $\pm S$.E.M., $n=2$ ). The ratios determined using the signals from the methyl group protons were usually higher than the ratios determined using the signals from the hydroxyethyl group protons.

Literature data indicate that acrylic and methacrylic monomers generally react randomly during a radical polymerization [16]. Reactivity ratios

lable 2. Price-Alfrey data for AA and HEMA

\begin{tabular}{lcr}
\hline & \multicolumn{1}{c}{ HEMA } \\
\hline AA & 0.83 & 0.88 \\
HEMA & 1.78 & -0.39 \\
\hline
\end{tabular}

Data from Ref. 16. 


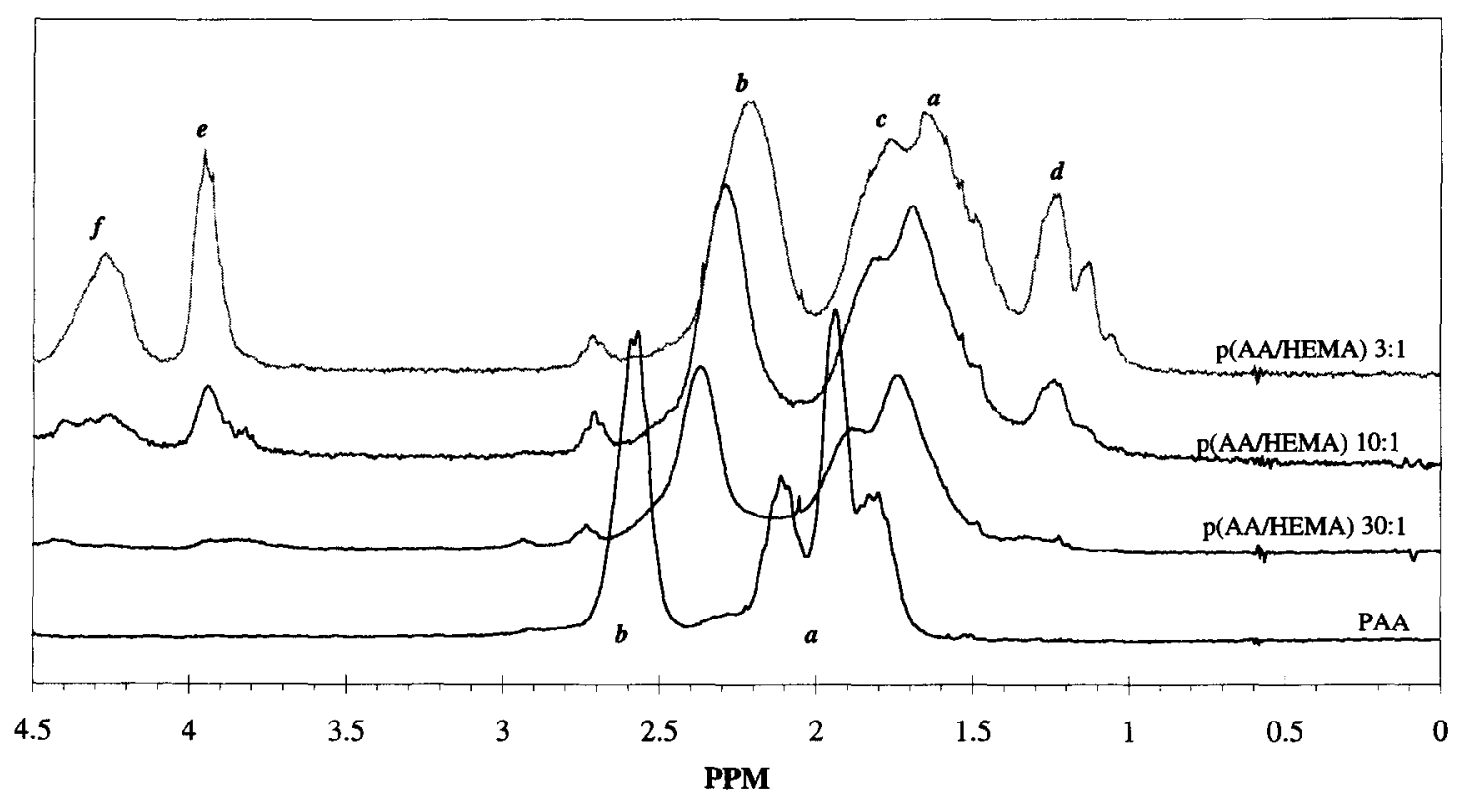

Fig. 1. 'H-NMR spectra for PAA and copolymers of AA and HEMA. $a-f$ refer to the peak assignments in Scheme 1.

for the AA/HEMA synthesis can be calculated from the Price-Alfrey equation:

$$
Q_{2}=\left(\frac{Q_{1}}{r_{1}}\right) * \exp \left[-e_{1}\left(e_{1}-e_{2}\right)\right]
$$

in which $Q$ and $e$ are measures of the reactivity and polarity, respectively. The Price-Alfrey $Q$ and $e$ values suggest that during a radical polymerization of these monomers a predominantly random monomer unit distribution will be obtained (see Table 2). Thus the values of $r_{1}$ (AA) and $r_{2}$ (HEMA) are 0.15 and 3.52 , respectively, showing that $\mathrm{AA}$ is more reactive towards both propagating species. Therefore the copolymer will have a distribution in composition with the HFMA units in a predominantly random placement $\left(r_{1} * r_{2}=0.52\right)$. This can also be concluded from the NMR spectra. It may be expected that in the first steps of the polymerization a product is formed with a higher AA content than the feed ratio. In the later steps more HEMA will be incorporated.

First, the minimal PEO molecular weight for detection of complex formation was determined using solutions of PEO with varying molecular weights as poly-base and PAA as poly-acid species. The number of hydrogen bonds formed upon complex formation is related to the change in $\mathrm{pH}$. A large change in $\mathbf{p H}$ denotes a large degree of complex formation. In Fig. 2 the $\mathrm{pH}$ is plotted as a function of the ratio PFO/PAA in molar concentration of monomer residues. The figure shows that the complex formation is related to the PEO molecular weight, i.e. the initial differential $\mathrm{pH}$ increase is increasing with the PEO molecular weight. It can be concluded that PEOs with a molecular weight higher than 6000 are

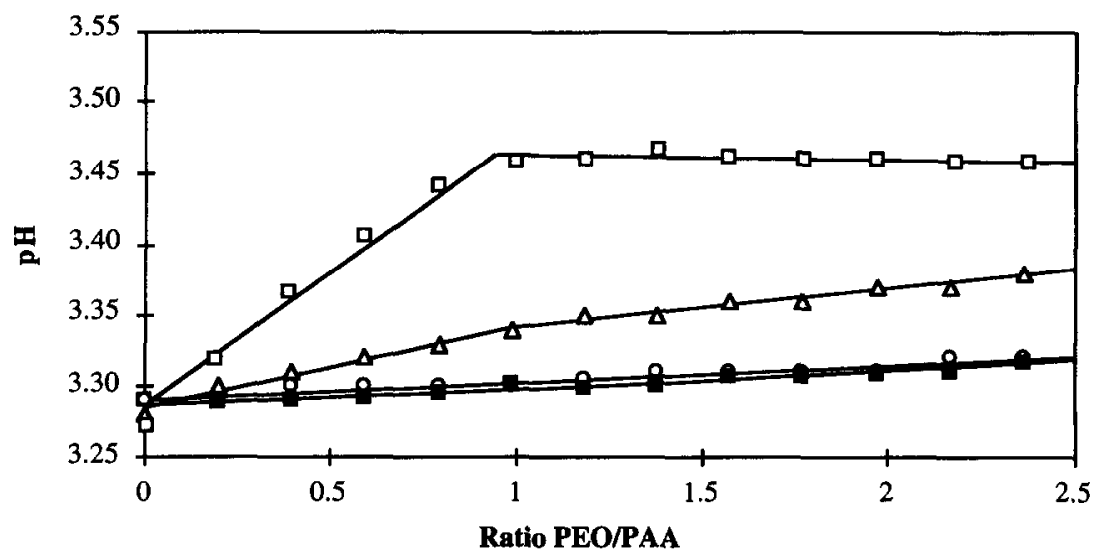

Fig. 2. Potentiometric titration of a $0.02 \mathrm{M}$ PAA solution with PEO solutions using PEO with different molecular weights: 1 , PEO-1; $\bigcirc$, PEO-3.4; $\triangle$, PEO-6; $\square$, PEO-25. Molar ratio based on monomer units EO and AA. 


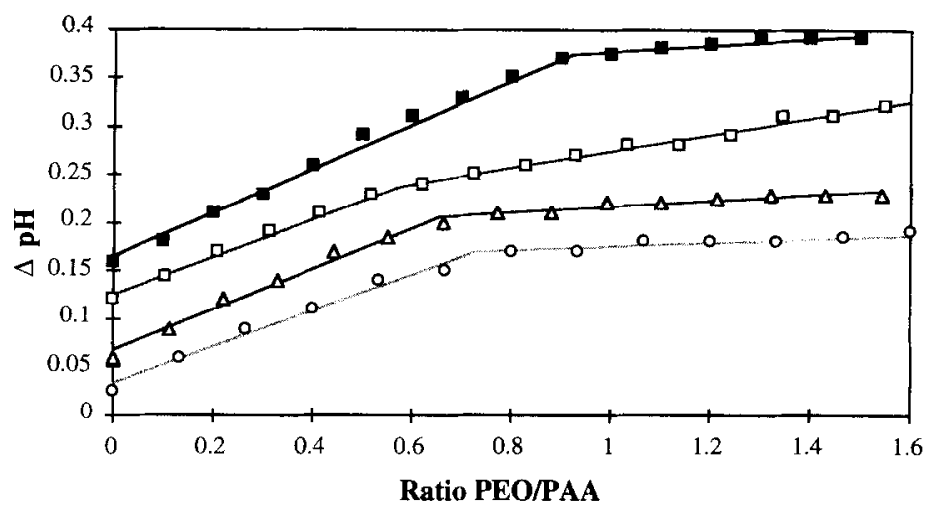

Fig. 3. Potentiometric titration of poly-acids with $\mathrm{PEO}-25$ as a function of the amount of PEO present (initial pH in parentheses): $\square$, PAA (3.41); $\square$, P(AA-co-HEMA) $30: 1$ (3.42); $\triangle$, P(AA-co-HEMA) 10:1 (3.2I); O, P(AA-co-HEMA) 3:1 (3.42), concentration 0.02 M (AA + HEMA). Molar ratio is based on monomer units EO and AA. Note that the curves have been shifted to avoid overlap.

required to detect complex formation using potentiometric titration. This is in agreement with the literature [17], where a minimum molecular weight of 8000 for PEO is found to detect complex formation in the PAA/PEO system. For optimal sensitivity PEO-25 was further used in the copolymer titrations.

The influence of HEMA comonomer units in the acrylic polymer on the complex formation with PEO was investigated using (co)polymers with different molar ratios of HEMA and AA. The results of the potentiometric titrations are shown in Fig. 3. To obtain an impression about the degree of complexation in terms of fraction of carboxylic acid groups associated with ether oxygens, $\mathrm{pH}$ values can be converted to the degree of complexation, $\Theta$, by considering the equilibria involved in the intermacromolecular hydrogen bonding and the intramolecular dissociation of the polyacid [18].

$$
\Theta=1-\left(\frac{10^{-\rho \mathrm{H}}}{10^{-\mathrm{pH}_{\mathrm{l}}}}\right)^{2} .
$$

Figure 4 shows the degree of complexation as a function of the ratio of PEO and P(AA-CO-HEMA).

Initially, all curves (in both Figs 3 and 4) show a constant slope until a certain point where the slope decreases. The monomer ratio at this cross-over point is about unity for the homopolymer PAA and about 0.6 for the copolymers. The cross-over ratio is also dependent on the amount of HEMA incorporated in the acrylic copolymer: more incorporated HEMA residues leads to a higher cross-over ratio at an approximately constant degrec of complexation $\Theta$ at this point.

In the interpretation of the results a clear difference should be made between the monomer ratio and the degree of complexation. The monomer ratio of PEO and the acrylic polymer shows only the amounts of the polymers in solution, whereas the degree of complexation, $\Theta$, is an indication of the number of carboxylic acid groups taking part in complexation. $\Lambda$ high monomer ratio at a certain value of $\Theta$ can be envisaged as an inefficient complexation of the carboxylic acid groups with $\mathrm{PEO}$, i.e. a relatively high amount of PEO is needed to interact with the number of carboxylic acid groups indicated by $\Theta$. Thus, in copolymers containing an increasing amount of HEM $\Lambda$ an increasing amount of PEO is required to reach an equilibrium complexation $(\Theta=0.43$ and $r=0.57$ for P(AA-Co-HEMA)-30; $\Theta=0.49$ and $r=0.62$ for $\mathrm{P}(\mathrm{AA}-\mathrm{Co}$-HEMA)-10; and $\Theta=0.49$ and

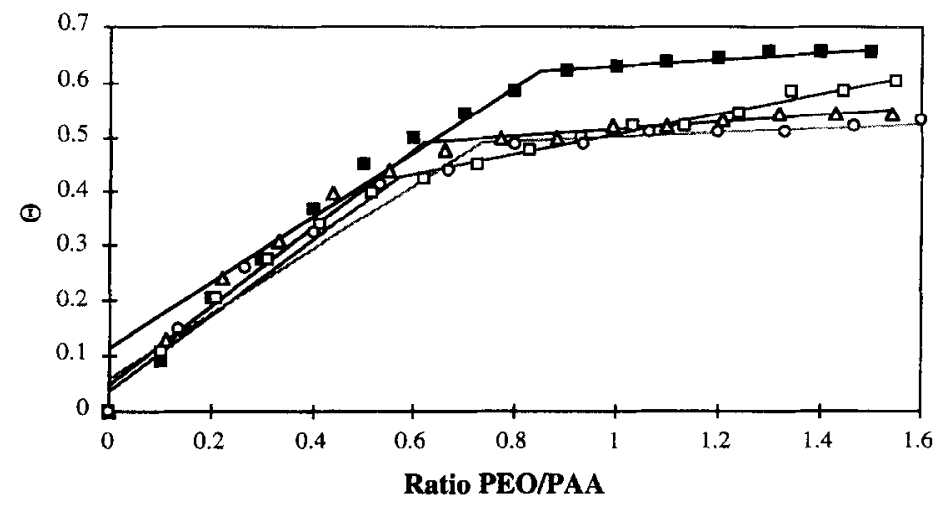

Fig. 4. Degree of complexation $\Theta$ for different poly-acids as a function of the amount of PEO-25 present:

口, PAA; $\square$, P(AA-co-HEMA) $30: 1 ; \triangle$, P(AA-co-HEMA) $10: 1 ; O, P(A A-c o-H E M A) ~ 3: 1$, concentration $0.02 \mathrm{M}$ (AA + HEMA). Molar ratio is based on monomer units EO and AA. 


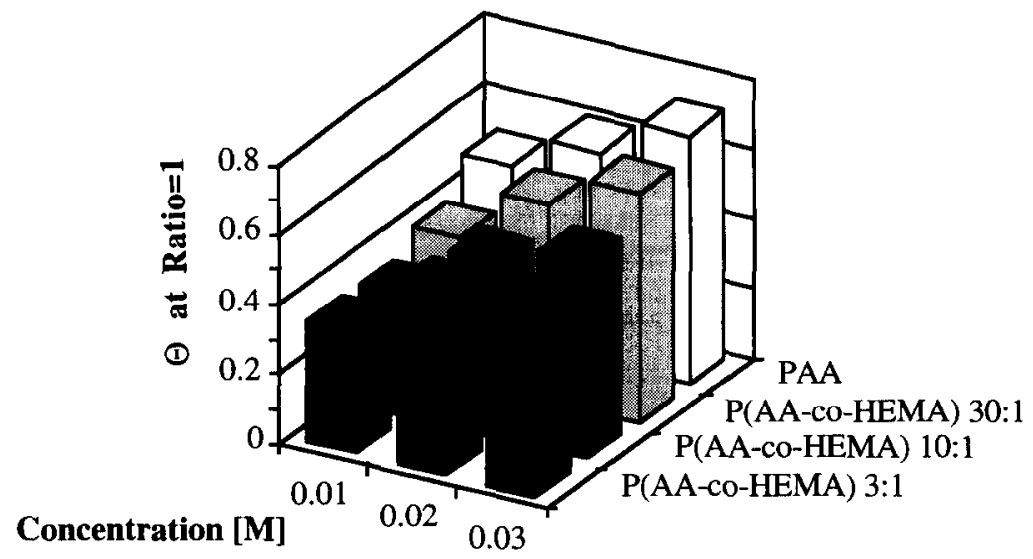

Fig. 5. Degree of complexation $\Theta$ for PAA and copolymers of AA and HEMA with PFO at unity ratio of EO/AA units. Concentration in monomer units (AA + HEMA).

$r=0.73$ for $\mathrm{P}$ (AA-co-HEMA)-3 at cross-over, $r$ is monomer ratio PEO/PAA).

A model study using Courtauld atomic models showed that it is possible to form an $\mathrm{H}$-bond between the carboxylic acid groups of PAA and the ether groups of PEO when the PEO molecule forms a helix around the PAA molecule. By introducing structure defects like HEMA units this conformation for the PEO is hindered and therefore complex formation is less efficient. Thus more PEO is required to interact with a certain number of carboxylic acid groups (in terms of Fig. 4, a higher monomer ratio PEO/PAA $(r)$ is required to reach a certain value of $\Theta$ ).

Iliopoulos et al. $[11,13]$ found a minimum blocklength of seven acrylic acid units to be necessary for any complexation to occur, based on measurements using partially neutralized PAA and PEO. In the current copolymers the probability of the formation of a block containing seven or more acrylic acid units in the case of a 3:1 AA:HEMA monomer feed is $13 \%$. However, a minimum of $30 \%$ of the AA units is involved in intermacromolecular hydrogen bonding between the copolymers and PEO $(\Theta$ always $>0.3$ in Fig. 5). This is more than twice the amount found in the system using a partially neutralized PAA. This may be explained when the type of structure defect is taken into account. In the latter system the structure defects are negatively charged acrylate groups which impede physical interaction with any approaching nucleophilic PEO chain. The charged AA groups may also move along the polymer chain, giving a spatially delocalized effect. In our case the structure defects are uncharged HEMA residues, which may even have a slight interaction with PEO.

This effect was also found in the literature [19], where the complexation of PEO (molecular weight 42,000 ) with copolymers of AA and $N$-isopropylacrylamide was studied. It was found that for the uncharged copolymers a higher degree of complexation was detected than for charged copolymers with the same degree of substitution. It was postulated that the charge caused the poly-acrylic chain to stretch, thus preventing interaction with the PEO chain.
Figure 5 shows that the degree of complexation $(\Theta)$ is dependent on the number of HEMA residues present. The PAA polymer shows a $\Theta$ of $71 \%$ (for $0.03 \mathrm{M}$ concentration), whereas the copolymers show a lower degree of complexation $(\Theta, 66 \%$ for the $30: 1$ copolymer, in which case the probability of formation of a block of seven AA residues is $79 \% ; 56 \%$ for the $10: 1$ copolymer with a probability of $51 \%$; and $52 \%$ for the $3: 1$ copolymer with a probability of $13 \%$ ). Thus, it may be concluded that a small number of structure defects in the PAA chain decreases the number of carboxylic acid groups taking part in complexation, but not as much as can be expected from the probability of the presence of a block of seven AA units. For a greater amount of structure defects no complex formation was detectable using potentiometric and viscosimetric methods. This was shown for AA/HEMA copolymers containing an excess of HEMA residues (copolymers with AA/HEMA ratios of $1: 1,1: 3$ or $1: 10)$ [20].

\section{CONCLUSIONS}

By introducing non-ionic structure defects in a poly-acid, complex formation with a poly-base-like PEO is quantitatively restricted. The degree of restriction depends on the number of structure defects. Furthermore, the type of structure defect plays a paramount role in the non-complexing behaviour.

Increasing the number of HEMA structure defects in a poly(acrylic acid) results in a decreased degree of complexation $\Theta$ of the carboxylic acid groups. When comparing the amount of $P E O$ required to reach an equilibrium complexation (the cross-over ratio) it can be seen that an increasing number of HEMA structure defects results in a less efficient packing of PEO on the acrylic acid copolymer chain. The degrees of complexation are much higher than the theoretical ones based on the probability of the formation of a sequence of seven $\mathrm{AA}$ residues, as found by Iliopoulos [11] for negatively charged carboxylate structure defects. The extra repulsion effect in the ionized polymers may be induced by the movement of the charged AA groups along the polymer chain. 


\section{REFERENCES}

1. K. L. Smith, A. E. Winslow and D. E. Petersen. Ind. Engng Chem. 51, I361 (1959).

2. F. E. Bailey Jr, R. D. Lundberg and R. W. Callard. J. Polym. Sci. Part A, Polym. Chem. 2, 845 (1964).

3. H. L. Chen and H. Morawetz. Eur. Polym. J. 19, 923 (1983).

4. E. Tsuchida, Y. Osada and H. J. Ohno. Macromol. Sci.-Phys. B17, 683 (1980).

5. E. A. Bekturov and L. A. Bimendina. Adv. Polym. Sci. 41, 99 (1981).

6. E. A. Bekturov, L. A. Bimendina and S. S. Saltybaeva. Makromol. Chem. 180, 1813 (1979).

7. L. A. Bimendina and A. A. Kurmanbaeva. Polym. Bull. 11, 557 (1984)

8. K. Abe and S. J. Senoh. Polym. Sci. Part A, Polym. Chem. 24, 3461 (1986).

9. L. A. Bimendina, V. V. Roganov and E. A. Bekturov. J. Polym. Sci., Polym. Symp. 44, 65 (1974)
10. T. Ikawa, K. Abe, K. Honda and E. Tsuchida. J. Polym. Sci. Polvm. Chem. Edn 13, 1505 (1975).

11. I. Iliopoulos and R. Audebert. Polym. Bull. 13, 171 (1985).

12. I. Iliopoulos, J. L. Halary and R. Audebert. J. Polym. Sci. Part A. Polym. Chem. 26, 275 (1988).

13. I. Iliopoulos and R. Audebert. Eur. Polym. J. 24, 171 (1988).

14. V. Baranovsky, S. Shenkov, I. Rashkov and G. Borisov. Eur. Polym. J. 28, 475 (1992).

15. M. Macret and G. Hild. Polymer 23, 81 (1982).

16. J. Brandrup and E. H. Immergut. Polymer Handhook, 3rd edn. Wiley, New York ([989).

17. V. Baranovsky, S. Shenkov, I. Rashkov and G. Borisov. Eur. Polym. J. 27, 189 (1991).

18. Y. Osada and M. Sato. J. Polym. Sci. Polym. Lett. Edn 14, 129 (1976).

19. G. Bokias, G. Staikos, I. Iliopoulos and R. Audebert. Macromolecules 27, 427 (1994).

20. M. J. Krupers, unpublished work. 